\title{
Comparative Evaluation of Baska Mask and LMA Supreme in Patients Undergoing Short Surgical Procedures Under General Anaesthesia
}

\author{
Neeta Verma ${ }^{1}$, Ankita Nigam² ${ }^{2}$ Amol Singam ${ }^{3}$ \\ ${ }^{1}$ Department of Anaesthesiology, Jawaharlal Nehru Medial College, Sawangi, Meghe, Wardha, Maharashtra, India. \\ ${ }^{2}$ Department of Anaesthesiology, Jawaharlal Nehru Medial College, Sawangi, Meghe, Wardha, Maharashtra, India. \\ ${ }^{3}$ Department of Anaesthesiology, Jawaharlal Nehru Medial College, Sawangi, Meghe, Wardha, Maharashtra, India.
}

\section{ABSTRACT}

\section{BACKGROUND}

Supraglottic airway devices (SAD) are used for airway management in patients undergoing general anaesthesia, averting the need of tracheal intubation. Laryngeal mask airway is easy to insert and can be inserted by paramedics also in case of emergency. Here we primarily compared the efficacy of Baska Mask and LMA supreme in terms of ease of insertion. The secondary outcome was assessment of oropharyngeal seal pressure and post op laryngopharyngeal complications.

\section{METHODS}

A sample size of 30 was calculated in each group. Patients were divided into 2 groups, Group B and Group S. After giving premedication, Anaesthesia was induced with propofol $3 \mathrm{mg} / \mathrm{Kg}$ and anaesthetic depth will be deepened with $2 \%$ sevoflurane in oxygen using bag mask ventilation. An appropriately sized prior lubricated Baska mask and LMA supreme was inserted in Group B and Group S, respectively and ease of insertion was assessed.

\section{RESULTS}

The BM was successfully inserted in 23 patients $(76.6 \%)$ in the first attempt and LMA supreme was successfully inserted in 29 patients $(96.6 \%)$ in the first attempt. The difference was found to be statistically significant $(\mathrm{P}=0.028)$. BM created a significantly higher oropharyngeal seal pressure than the LMA supreme group $(\mathrm{P}<$ 0.001). No significant difference in postoperative laryngopharyngeal complications was observed.

\section{CONCLUSIONS}

From the present study, it is concluded that LMA supreme is easier to insert than BM. The BM creates higher oropharyngeal seal pressure and thus provides a better airway seal than the LMA supreme. The incidence of postoperative laryngopharyngeal complications is similar in both the groups.

\section{KEY WORDS}

Baska Mask, LMA Supreme, Oropharyngeal Seal Pressure, Efficacy
Corresponding Author:

Ankita Nigam,

T-19, Shalinata Bai PG Girls Hostel,

JLN, Wardha, Maharashtra, India.

E-mail: ankitanigam95@gmail.com

DOI: $10.14260 /$ jemds/2020/515

How to Cite This Article:

Verma N, Nigam A, Singam A. Comparative evaluation of Baska mask and LMA supreme in patients undergoing short surgical procedures under general anaesthesia. J Evolution Med Dent Sci 2020;9(33):2377-2380, DOI:
Submission 16-04-2020,

Peer Review 08-07-2020,

Acceptance 14-07-2020,

Published 17-08-2020.

Copyright (C) 2020 JEMDS. This is an open access article distributed under Creative Commons Attribution License [Attribution 4.0 International (CC BY 4.0)] 


\section{BACKGROUND}

Supraglottic Airway Devices (SADs) are used for airway management in patients undergoing various surgical procedures under general anaesthesia averting the need of tracheal intubation. They offer an excellent non-invasive option for ventilation instead of the endotracheal tube (ET) and the face mask. The use of SADs has been increasing in the past decade for the ease of insertion and insignificant complications. SADs are mostly useful in emergency and critical situations, providing a rapid access to the airway. They also help in providing a rescue access in patients with difficult intubation.

SAD can be used safely in variety of age groups and in various surgical procedures. It has the advantage of being minimally invasive, less traumatic, and having a relatively effortless insertion without muscle relaxants or laryngoscopy. The ensuing airway stimulation is less, resulting in minimal hemodynamic changes, less coughing and agitation on awakening from anesthesia, and less severe postoperative sore throat. ${ }^{1}$

The advantages of the SAD over ET include the ease of insertion, rapidity, low postoperative complications, and reduced autonomic imbalance during insertion. The SADs are positioned outside the larynx making them easy for use in certain clinical situations. The sealing site of the SAD varies from the base of the tongue and perilaryngeal sites. The advanced models of the SAD improve the efficacy of the ventilation and also a patient's safety.

The models of SAD differ in the sealing mechanisms and aspiration protection designs.

The SAD are broadly divided into the first-generation devices (only breathing lumen) and second-generation devices (additional lumen for aspiration of the gastric contents). ${ }^{2}$ The complications with the first-generation devices include the increased risk of aspiration and poor ventilation.

The Laryngeal mask airway supreme is a second generation gastric access device, which has a soft, elongated cuff designed to form an effective first seal with the oropharynx permitting higher glottic seal pressures and an innovative second seal with the upper oesophageal sphincter. This maintains the patency of the drain tube reducing the risk of insufflation during ventilation and the risk of regurgitated gastric contents leaking around the tip of the mask.

The Baska Mask designed by Australian anesthetists Kanag and Meena Baska, is a new CE--approved and internationally patented EAD, provided in single use and multi-use versions. The Baska mask obviates the need for an orogastric tube and replaces this with a sump and two drains. The Baska mask brings together features of: (1) the LMA-ProSeal i.e. high seal pressure, gastric access port and bite block, which facilitate ventilation, provide airway protection, and minimize airway obstruction, respectively; (2) the LMA-Supreme, i.e. oval shaped, anatomically curved airway tube which incorporates a gastric drain tube (3) the i-gel i.e. a gel-like cuff instead of an inflatable balloon; and (4) the Slipa i.e. a cuffless, anatomically pre-shaped sealer with a sump reservoir. The last three have an incorporated bite block to protect the airway. ${ }^{3}$ The Baska Mask presents with a bite block throughout the entire length of the airway tube. The oval-shaped airway tube matches the shape of the mouth and reduces rotation within the pharynx. Here we will compare the clinical efficacy of Baska mask and LMA supreme on various aspects. The primary outcome was ease of insertion and secondary outcome were to compare oropharyngeal seal pressure and postoperative complications.

\section{METHODS}

This study was conducted in the department of Anaesthesia at Jawaharlal Institute of Medical Sciences, Sawangi (Meghe) Wardha in accordance with the guidelines and permission of the institutional ethical review board. Study design was prospective comparative study. The patients included for the study was between the age group of 18 to 60 years of both sex and ASA grade I/II posted for elective surgery. The exclusion criteria included was Patients not willing to undergo surgery under G.A. Patients with anticipated difficult tracheal intubation, Pregnant and nursing mothers, Patients with significant cardiovascular, renal, hepatic or respiratory disorders.

Sample was derived using software openepi.com. Assuming ease of insertion among LMA supreme group to be $97.1 \%$, keeping power at $80 \%$ and two-sided confidence level of $95 \%$ ( $\alpha$ error at 0.05 a sample of 26 patients were required to detect ease of insertion between two groups. We included 30 patients in each group to compensate for possible dropouts. ${ }^{4}$

There were total 60 patients in the study. All the Patients were Randomly Divided into Two Equal Groups of 30 Patients each by simple randomization method. GROUP B in which Baska mask was inserted and group $\mathrm{S}$ in which LMA supreme was inserted. All patients who matched the inclusion criteria were assessed by a pre-anaesthesia examination.

Baseline measurements of blood pressure, pulse rate, arterial oxygen saturation was taken.

Group B- Baska Mask Group; Group S- LMA Supreme Group all patients were kept NBM for 6 hrs., for solids and 2 hrs., for clear fluids prior to the surgery. Inside the OT, multiparameter monitors were attached and routine parameters like PR, ECG, NIBP, SPO2, and ETCO2 were monitored. A peripheral intravenous line were secured in the upper limb with an appropriate infusion. Patients were preoxygenated with $100 \%$ oxygen for $3 \mathrm{~min}$. Anaesthesia was induced with propofol 3 $\mathrm{mg} / \mathrm{Kg}$ and anaesthetic depth will be deepened with $2 \%$ sevoflurane in oxygen using bag mask ventilation. Patient were kept in sniffing position prior to device insertion. If required, an additional dose of propofol wi used to achieve adequate depth of anaesthesia prior to the device insertion. Complete jaw relaxation was there to confirm adequate depth. Then, an appropriate sized prior lubricated Baska Mask and LMA Supreme was inserted in Group B and Group S, respectively. Maximum attempts was restricted to three, failing to it appropriate sized ETT will be used to manage the patient. The number of insertion-attempts and time for insertion (interval from when the supraglottic device came into contact with the patient's lip to the first square wave on the capnogram with adequate ventilation) were recorded. The ease of insertion of SGA was subjectively assessed on a 2-point scale (1=easy, 2= difficult) The insertion was considered EASY if it was successfully inserted in a single attempt and it was considered as difficult when more than one attempt was 
required for successful placement. Anaesthesia was maintained with oxygen, nitrous oxide, and sevoflurane.

Proper placement of the inserted device was confirmed by square wave capnography, normal chest movement and auscultation of breath sounds in front of neck of the patient. Inadequate chest rise, abnormal capnogram, drop in $\mathrm{SpO}_{2}$ \& lt; $95 \%$ will mean an improper positioning of the device. Nasogastric tube was inserted in both the device.

The Oropharyngeal sealing pressure (OSP) was measured using a fresh gas flow rate of $5 \mathrm{~L} / \mathrm{min}$, closure of the adjustable pressure limiting valve of the anaesthetic circuit and recording the pressure when gas was heard leaking around the device.

Then, trachea was continuously auscultated for an audible leak, while airway pressure will be monitored from the pressure gauge of the anaesthesia machine. A maximum of 40 $\mathrm{cm} \mathrm{H}_{2} \mathrm{O}$ of airway pressure was allowed during the leak check procedure.

At the end of surgery all anaesthetics was tapered off. Once the patient was wide awake and responsive, the device was removed. The relevant postoperative complications observed after extubation include cough, local trauma, dysphonia, dysphagia, postoperative nausea and vomiting (PONV), and presence of gastric fluid in the oral cavity.

\section{Statistical Analysis}

Data was statistically analysed. Student $t$ test has been used to find the significance of study parameters on continuous scales, between 2 groups on metric parameters. Chi square test was done to find the significance of study parameters between 2 groups on categorical scale.

\section{RESULTS}

A total of 60 participants were included in the study in which 30 were in group B and 30 were in group S. There was no statistically significant difference between the two groups in age, height, weight, BMI (table 1) and American society of anesthesiologists (ASA) grade.

The BM was successfully inserted in 23 patients $(76.6 \%)$ in the first attempt and LMA supreme was successfully inserted in 29 patients (96.6\%) in the first attempt. The difference was found to be statistically significant $(\mathrm{P}=0.028)$

The mean oropharyngeal seal pressure in the $\mathrm{B}$ group was $33.25 \pm 6.78 \mathrm{cmH}_{2} \mathrm{O}$ and that of the $\mathrm{S}$ group is $27.45 \pm 2.34$ $\mathrm{cmH}_{2} \mathrm{O}$. BM created a significantly higher oropharyngeal seal pressure than the LMA supreme group

$(p<0.001)$. The maximum oropharyngeal seal pressure of $40 \mathrm{~cm}$ of water was achieved in 8 patients $(26.6 \%)$ in the BM group. Gastric regurgitation was not observed in both the groups. 2 patients $(6.6 \%)$ in BM group had transient cough on removal of device. Blood staining on mask removal was observed in one patient (3.3\%) in BM group and two patients $(6.6 \%)$ in LMA supreme group. There was no significant difference in two groups in terms of these complications. There was no incidence of vomiting, regurgitation and lip/dental trauma in both the groups. Sore throat was observed in $4(13 \%)$ patients in group B and $2(6.6 \%)$ in group $\mathrm{S}$ within $24 \mathrm{hr}$ postoperatively. Hoarseness of voice, dysphonia, dysphagia was not observed. Nasogastric tube can be easily inserted in both the groups.

\begin{tabular}{|cccc|}
\hline Parameter & Group B & Group S & P Value \\
Age (years) Mean \pm SD & $40.65 \pm 4.52$ & $45.98 \pm 15.77$ & 0.144 \\
Gender (male/female) & $18 / 12$ & $20 / 10$ & 0.101 \\
Height $(\mathrm{cm})$ Mean \pm SD & $161.12 \pm 8.94$ & $160.10 \pm 8.01$ & 0.617 \\
Weight $(\mathrm{kg})$ Mean \pm SD & $58.52 \pm 10.30$ & $60.28 \pm 9.67$ & 0.137 \\
\hline \multicolumn{4}{|c|}{ Table 1. Patient Characteristics } \\
\hline \multicolumn{4}{|c}{} \\
\hline
\end{tabular}

\begin{tabular}{|ccc|}
\hline Successful Insertion & Group B & Group S \\
Easy & $23(76.6 \%)$ & $29(96.6 \%)$ \\
Difficult & $4(13.3 \%)$ & $1(3.3 \%)$ \\
\hline \multicolumn{3}{|c}{ Table 2. Ease of Insertion } \\
\hline
\end{tabular}

\begin{tabular}{|ccc|}
\hline Group B (Mean \pm SD) & Group S (Mean \pm SD) & P Value \\
$33.25 \pm 6.78 \mathrm{cmH}_{2} \mathrm{O}$ & $27.45 \pm 2.34 \mathrm{cmH}_{2} \mathrm{O}$ & $\mathrm{p}<0.001$ \\
\hline \multicolumn{2}{|c|}{ Table 3. Oropharyngeal Seal Pressure } \\
\hline
\end{tabular}

\begin{tabular}{|ccc|}
\hline Complication & Group B & Group S \\
Transient cough on removal & $2(6.6 \%)$ & 0 \\
Blood stain on removal & $1(3.3 \%)$ & $2(6.6 \%)$ \\
Sore throat & $4(15 \%)$ & $2(6.6 \%)$ \\
PONV & 0 & 0 \\
Dysphagia/dysphonia/hoarseness & 0 & 0 \\
Lip/dental trauma & 0 & 0 \\
\hline Table 4. Postoperative Complications \\
\hline
\end{tabular}

\section{DISCUSSION}

In this study, placement of LMA successfully in first attempt was significantly higher in group $S$ that is with LMA supreme group. In previous study done by. Alexiev V, Ochana A, Abdelrahman D, Coyne J, McDonnell J,O'Toole D, et al. BM had shown significantly less insertion success rate than LMA classic in female patient undergoing surgery (73\% verses $98 \%$ respectively, $\mathrm{P}<0.001)^{5}$. However, in a study conducted by Aziz ARA, Osman YM, when the BM airway was compared with Igel for controlled ventilation in obese patients undergoing ambulatory surgery, both the devices were easily inserted with high success rates $76.67 \%$ versus $73.3 \%$, no statistically significant difference) Successful insertion time was significantly shorter in igel group. ${ }^{6}$

Similarly in a study done by Al-Rawahi SAS, Aziz H, Malik AM, Khan RM, Kaul N. When BM was compared with LMA Proseal in adults, insertion time was short in BM group (16.43 \pm 4.54 versus $21.45 \pm 6.13 \mathrm{~s}, \mathrm{p}=0.001$ ) and no difference in no. of attempts required for placement of device in both the groups. $^{7}$

When. Anand LK, Goel N compared, LMA Proseal with LMA supreme in patients undergoing cholecystectomy, ease of insertion was higher in LMA supreme group. ${ }^{7}$ time of insertion was also shorter LMA supreme group. $8,9,10$

When in a study conducted by Park SY, Rim JC LMA supreme was compared to I-GEL, no significant difference in insertion time, no. of insertion attempts and rate of insertion..$^{11,12,13}$

The secondary outcome of this study was to assess the oropharyngeal seal pressure created by both the devices, more the seal pressure better the seal between artificial airway and oropharynx. Here group B had significantly higher seal pressure as compared to group $\mathrm{S},(\mathrm{p}<0.001)$. All the patents in group $\mathrm{S}$ had seal pressure less than $35 \mathrm{cmH}_{2} \mathrm{O}$ and more than 
$35 \mathrm{cmH}_{2} \mathrm{O}$ in group B in $40 \%$ patients. In previous studies findings were similar, An initial observational study of the BM in low risk female patients done by Alexiev V, Salim A, also found a mean airway leak pressure of $35.7 \pm 13.3 \mathrm{~cm} \mathrm{H} \mathrm{H}_{2} \mathrm{O}^{3}$ Another study conducted by Shanmugavelu G, Kanagarajan T., The oropharyngeal seal pressure was significantly higher in the BM than the i-gel for controlled ventilation in obese patients and in laparoscopic surgeries. ${ }^{14}$ The mean oropharyngeal leak pressure was significantly lower in the LMAS group than in LMA ProSeal during laparoscopic cholecystectomy. ${ }^{8}$

The postoperative complications were also similar in both the groups, no significant difference was there. The postoperative complications were not significantly different when LMAS was compared with LMA classic, I gel and LMA ProSeal. ${ }^{8}$

\section{CONCLUSIONS}

LMA supreme is easier to insert than BM. BM creates higher oropharyngeal seal pressure and thus provides a better airway seal than the LMA supreme. The incidence of postoperative laryngopharyngeal complications is similar in both the groups. The gastric port was correctly positioned over the oesophagus in both the devices reducing the chances of aspiration.

Financial or Other Competing Interests: None.

\section{REFERENCES}

[1] Udaybhaskar V, Singam A, Dodeja H, et al. Comparison of inhalational vital capacity induction with sevoflurane to intravenous induction with propofol for insertion of laryngeal mask airway in adults: a randomized study. Anesth Essays Res 2018;12(1):73-9.

[2] Timmermann A, Bergner UA, Russo SG. Laryngeal mask airway indications: new frontiers for second-generation supraglottic airways. Curr Opin Anaesthesiol 2015;28(6):717-26.

[3] Alexiev V, Salim A, Kevin LG, et al. An observational study of the Baska ${ }^{\circledR}$ mask: a novel supraglottic airway. Anaesthesia 2012;67(6):640-5.
[4] Jayalekshmi S, Paul C, Thomas MK. Efficacy of Baska mask and Laryngeal mask airway supreme during positive pressure ventilation - a comparative study. J Anaesthesiol Clin Pharmacol 2020;36(1):31-6.

[5] Alexiev V, Ochana A, Abdelrahman D, et al. Comparison of the Baska mask with the single-use laryngeal mask airway in low-risk female patients undergoing ambulatory surgery. Anaesthesia 2013;68(10):1026-32.

[6] Aziz ARA, Osman YM. Comparison of I-gel with Baska mask airway for controlled ventilation in obese patients undergoing ambulatory surgery: a prospective randomized trial. J Anaesthesiol 2017;5(4):29-35.

[7] Al-Rawahi SAS, Aziz H, Malik AM, et al. A comparative analysis of the Baska mask vs. Proseal laryngeal mask for general anesthesia with IPPV. Anaesth Pain Intensive Care 2013;17(3):233-6.

[8] Anand LK, Goel N, Singh M, et al. Comparison of the supreme and the ProSeal laryngeal mask airway in patients undergoing laparoscopic cholecystectomy: a randomized controlled trial. Acta Anaesthesiol Taiwan 2016;54(2):44-50.

[9] Hoşten T, Yildiz TŞ, Kuş A, et al. Comparison of Supreme laryngeal mask airway and ProSeal laryngeal mask airway during cholecystectomy. Balkan Med J 2012;29(3):314-9.

[10] Gill RK, Tarat A, Pathak D, et al. Comparative study of two laryngeal mask airways: proseal laryngeal mask airway and Supreme laryngeal mask airway in anesthetized paralyzed adults undergoing elective surgery. Anesth Essays Res 2017;11(1):23-7.

[11] Radhika KS, Sripriya R, Ravishankar M, et al. Assessment of suitability of i-gel and laryngeal mask airway-supreme for controlled ventilation in anesthetized paralyzed patients: a prospective randomized trial. Anesth Essays Res 2016;10(1):88-93.

[12] Park SY, Rim JC, Kim H, et al. Comparison of i-gel@ and LMA supreme ${ }^{\circledR}$ during laparoscopic cholecystectomy. Korean J Anesthesiol 2015;68(5):455-61.

[13] Teoh WHL, Lee KM, Suhitharan T, et al. Comparison of the LMA Supreme vs the i-gel in paralysed patients undergoing gynaecological laparoscopic surgery with controlled ventilation. Anaesthesia 2010;65(12):1173-9.

[14] Shanmugavelu G, Kanagarajan T. Comparing the functional analysis of i-gel with Baska mask in laparoscopic surgeries: an observational study. Int J Res Med Sci 2018;6(4):1440-3. 